\title{
15 Klimasensible Stadtplanung und Stadtentwicklung
}

\section{Judith Schröder und Susanne Moebus}

C. Günster ||. Klauber | B.P. Robra |C. Schmuker | A. Schneider (Hrsg.) Versorgungs-Report Klima und Gesundheit. DOI 10.32745/9783954666270-15, (C MWV Medizinisch Wissenschaftliche Verlagsgesellschaft Berlin 2021

Der vorliegende Beitrag spürt der Frage nach, wie sich Städte durch innovative Stadtplanung besser an den Klimawandel anpassen und wie Stadtplanung Gesundheitsressourcen stärken und -risiken mindern kann. Ausgehend von der industriellen Revolution als gemeinsamer historischer Wurzel für bestehende Herausforderungen durch Klimawandel und Verstädterung und deren Implikationen für Gesundheit, wird für einen Paradigmenwechsel plädiert, der Gesundheit nicht nur unter Gesichtspunkten der Krankheitsbekämpfung begreift, sondern auf die salutogenen Faktoren von Stadtentwicklung unter der notwendigen Berücksichtigung von Klimaschutz- und Klimaanpassungsmaßnahmen fokussiert.

This article explores how cities can better adapt to climate change and how cities can better address health risks and chances through innovative urban planning. Starting from the industrial revolution as a common historical root for existing challenges of climate change and urbanization and their implications for health, the paper argues for a paradigm shift that understands health not only from the perspective of disease control but also from the salutogenic factors of urban development, taking into account the need for climate protection and adaptation measures.

\subsection{Gesundheit und die Megatrends des 21. Jahrhunderts}

\subsubsection{Der historische Zusammenhang zwischen Stadt, Gesundheit und Klimawandel}

Nicht viele Themen haben eine vergleichbare Zunahme an politischer Relevanz zu verzeichnen wie der anthropogene Klimawandel. Eingebettet in die größere Debatte der ökologischen Krise in Gestalt von Biodiversitätsverlust, Ressourcenknappheit, Erschöpfung der ökologischen Tragekapazität, zählt die Frage nach dem klimagerechten Umbau unserer Gesellschaft zu den Megatrends des 21. Jahrhunderts. Seit der Veröffentlichung der vom Club of Rome in Auftrag gegebenen Studie The Limits to Growth (Meadows et al. 1972) ist die Frage zum Umgang mit Natur angesichts der Erkenntnis der Endlichkeit natürlicher Ressourcen zunehmend in den Gesichtskreis gesellschaftspolitischer Wahrnehmung gerückt. Angesichts der für das menschliche Überleben notwendigen Bedin- 
gung des permanenten Austausches und des Stoffwechsels mit Natur, ist es nur verständlich, dass dieser Problematik ein erhöhtes Maß an Aufmerksamkeit zukommt und sich zahlreiche gesellschaftliche Gruppen der Thematik annehmen. Denn die wohl einschneidendste Erkenntnis hinsichtlich der Ursachenforschung ist, dass Haupttreiber und -verursacher des Klimawandels der Mensch selbst ist.

Zwar fußen menschliche Existenz und gesellschaftliche Entwicklung seit jeher auf Austauschprozessen mit Natur, aber diese Prozesse haben seit der industriellen Revolution eine bis dahin ungekannte Dynamik in Form einer sich scheinbar perpetuierenden Beherrschung der Natur durch technischen Fortschritt als wichtigstem Motor ökonomischen Wachstums gewonnen. Vor allem die Extraktion und Nutzung fossiler Energieressourcen wie Kohle, Erdöl und Erdgas kann als Wendepunkt gesellschaftlicher Entwicklung betrachtet werden, da es damit gelang, den benötigten Energiebedarf von temporär kurzweiliger biologischer Produktivität, z.B. in Form von nur langsam nachwachsenden Rohstoffen wie Holz (Rosenbaum u. Mautz 2011: 399), abzukoppeln und so die Produktivität von Arbeit um ein Vielfaches zu steigern. Durch Extrahieren und Verbrennen fossiler Energieträger wurden geologische und ökologische Erdveränderungen sowie exponentielle Wachstumsprozesse in Gestalt von Bevölkerungs- und Wohlstandsexplosionen in Gang gesetzt. Oder anders ausgedrückt: „Der Beginn des fossilen Energiezeitalters war ausschlaggebend für die Akkumulationsdynamik und den Clobalisierungsprozess kapitalistischer Cesellschaften“ (Kautnek 2012: 8). Trotz der Innovationskraft und permanenten Steigerung der Naturbeherrschung während der letzten 200 Jahre scheinen die Grenzen dieser Entwicklungen überschritten. Seit einigen Jahrzehnten sind biophysikalische Nebenwirkungen des fossilen Energiesystems zu beobachten, die nachhaltigen Einfluss auf das Erdklima ausüben (IPCC 2014).

Neben den wirtschaftlichen Veränderungsprozessen verschob sich mit Beginn der Indus- trialisierung auch das räumliche Verhältnis von Stadt und Land. Die Zentralisierung wirtschaftlicher Produktion in Fabriken hatte zur Folge, dass große Bevölkerungsteile vom Land in die Stadt strömten, um sich dort als abhängige Lohnarbeiter anzusiedeln. Die Produktionssteigerung ermöglichte die Versorgung einer erhöhten Bevölkerungszahl. Die Kombination aus dem Sinken der bis dahin hohen Kindersterblichkeit und dem Anstieg der Lebenserwartung trieb das Wachstum der Städte weiter in die Höhe (Lorberg u. Simon 2020: 368). Die Fabrikarbeit und die Entstehung der bürgerlich-kapitalistischen Gesellschaftsordnung in den Städten war gekennzeichnet durch eine sich ausbreitende Verelendung ganzer Bevölkerungsgruppen:

„Niedrige Löhne, lange Arbeitszeiten, Wohnungsnot, keine Rückgriffmöglichkeiten auf alte Formen sozialer Sicherung sind die Faktoren, die sich in physischer und psychischer Verelendung der Arbeiter, ... niederschlugen. Die Entwicklung einer neuen Verelendung weiter Kreise insbesondere der städtischen Bevölkerung durch die Fabrikarbeit entsprach weder in materieller noch ideeller Hinsicht den Erwartungen, die das Bürgertum an die Durchsetzung der bürgerlich-kapitalistischen Cesellschaftsordnung geknüpft hatte.“ (Rodenstein 1988: 69)

Die schlechten Wohn- und Arbeitsverhältnisse zogen Krankheiten und Epidemien nach sich, sodass Überlegungen zur räumlichen Organisation und öffentlicher Gesundheitspflege (Public Health) unausweichlich wurden. Die Idee des Gesundheitsschutzes in städtebaulichen Leitkonzepten war dabei zwar nicht neu, fand sich dieser doch bereits im Sinne der Stadthygiene in Konzepten zur Trinkwasserversorgung und Abfallentsorgung antiker Stadtstaaten wieder. Dennoch erlangte die Problematik durch das enorme Bevölkerungswachstum in den Städten, die daraus folgende hohe bauliche Verdichtung und die schlechten hygienischen Lebensbedingungen im Zuge der Industrialisierung eine neue Qualität mit sozialen und politischen Implikationen, auf die es politisch und 
stadtplanerisch zu reagieren galt (Rodenstein 1988). Beispielhaft sei hier das stadtplanerische Konzept der Gartenstadt von Ebenezer Howard Ende des 19. Jahrhunderts genannt. Die Idee der Gartenstadt umfasste nicht nur einen Plan für die Entwicklung einer grünen Siedlung, mit kurzen Wegen zu zentralen Einrichtungen und Produktionsstätten, sondern auch einen Verwaltungs- und Finanzplan, der auf eine gerechte Ressourcenverteilung ausgerichtet war und auf die am stärksten gefährdeten Bevölkerungsgruppen wie „alte Arme“ abzielte (Howard 2010 [1989]). Langfristig und flächendeckend durchsetzen konnten sich solche Ansätze gegenüber wirtschaftlichen Interessen jedoch nicht, sodass die Folgen der industriellen Revolution und deren ökologischen und gesundheitlichen Schattenseiten in unseren Städten bis heute präsent sind.

Das sich in dieser Zeit entwickelnde Verständnis einer städtischen öffentlichen Gesundheit - oder auch Urban Public Health - ist nach dem zweiten Weltkrieg in weiten Teilen verloren gegangen und von einer kurativen Individualmedizin abgelöst worden. Mit der Debatte um die ökologische Krise in den 198oerJahren ist die Rolle der Stadtplanung für die Gestaltung und Verbesserung von Lebensverhältnissen und damit auch der Gesundheit wieder deutlich geworden (Rodenstein 1988).

\subsubsection{Urbane Gesundheit und die Große Transformation}

Diese skizzenhafte Rekonstruktion zeigt die historischen Wurzeln, aus denen die Notwendigkeit einer globalen Debatte über eine Nachhaltigkeitsrevolution erwachsen ist. Die Idee der nachhaltigen Entwicklung ist seit der ersten UN-Klimakonferenz in Rio de Janeiro 1992 in einem Prozess der Überführung in konkrete Zielvorgaben und Politiken der Umsetzung begriffen. Zu den Leitvorgaben zählen unter anderem die von den Vereinten Nationen formulierten Nachhaltigkeitsziele (Sustainable De- velopment Goals) sowie das Pariser Klimaabkommen. Der Wissenschaftliche Beirat der Bundesregierung Globale Umweltveränderungen (WBGU) entwirft in seinem 2011 veröffentlichten Gutachten eine Vision eines neuen $\mathrm{Ge}$ sellschaftsvertrages für eine Große Transformation (WBGU 2011) zur erfolgreichen Umsetzung einer nachhaltigen Entwicklung (Vereinte Nationen 2015). Damit einher geht die Notwendigkeit einer postfossilen Wirtschaftsweise und klimaverträglicher Wertschöpfung, sie betrifft Produktion, Konsummuster und Lebensstile in lokalem, regionalem und globalem Ausmaß. Urbane Räume und damit einhergehend sowohl die Stadt(-entwicklung) als auch die Gesundheitsförderung werden dabei als zentrales Transformationsfeld adressiert:

„Die seit der UN-Konferenz in Rio de Janeiro 1992 propagierte nachhaltige (Stadt-)Entwicklung setzte aufÖkologie und damit die Reduzierung des motorisierten Individualverkehrs in den Städten, die Aufwertung städtischer Freiräume und Klimaschutz sowie die Energiebilanz von Gebäuden. Eine nachhaltige städtebauliche Entwicklung wurde in $\$ 1$ des Baugesetzbuches als Grundsatz verankert und umfasste damit implizit auch gesundheitsrelevante Aspekte." (Baumgart 2018:30)

Weiteres Zeichen eines Paradigmenwechsels ist auch die 1986 von der WHO verabschiedete Ottawa-Charta als Ergebnis der ersten internationalen Konferenz zur Gesundheitsförderung. Das gesundheitspolitische Grundsatzdokument und Aktionsprogramm gilt als Rahmung zur Verbesserung von Gesundheit und Lebensqualität auch in einer globalen Perspektive. Der Abbau gesundheitlicher Ungleichheiten und die soziale Verteilung von Gesundheitschancen werden hier als dringlichste Aufgaben unter Anerkennung dessen, dass Gesundheit in der alltäglichen Lebensumwelt jenseits von Gesundheitsversorgung entsteht, formuliert. Eine Grundvoraussetzung für die Umsetzung der Charta ist der Health-in-all-policies-Ansatz der WHO, der die Verankerung und Umsetzung von Gesundheit in allen Politikfeldern erfordert. 
Die Ebene der Stadt ist für die politische Verankerung und Umsetzung von hervorzuhebender Bedeutung. In der Gestalt und der Gestaltung von Städten spiegeln sich die jeweiligen spezifischen sozialen, wirtschaftlichen, politischen, technischen und ökologischen Umstände einer Zeit wider und korrespondieren ebenso mit dem stetigen Wandel dieser Umstände. Der aktuell beobachtbare Urbanisierungstrend hat weltweit ein Niveau erreicht, das in dieser Form bisher unbekannt war. Zurzeit leben 55\% der Weltbevölkerung in Städten mit einer Bevölkerung von mindestens 300.000 . Bis 2050 sollen es Prognosen zufolge bereits 68\% sein. In Europa sind es aktuell bereits fast 75\% (Deutschland: 77,3\%), für 2050 werden $83,7 \%$ (Deutschland: 84,3\%) erwartet (United Nations 2019). Der WBCU spricht deshalb inzwischen vom 21. Jahrhundert als dem Jahrhundert der Städte (WBGU 2016). Dabei sind Städte Hauptverbraucher von Energie, drei Viertel der energiebedingten Treibhausgasemissionen werden in Städten verursacht und Städte beanspruchen mehr als die Hälfte des Siedlungswasserverbrauchs; bei steigender urbaner Siedlungs- und Verkehrsfläche (Rink u. Kabisch 2017: 243).

\subsection{Klimaschutz und Klimaanpassung in einer gesundheitsfördernden Stadtentwicklung}

Der Anstieg der Durchschnittstemperatur durch das weltweite Emissionsverhalten und zunehmende Treibhausgaskonzentration in der Atmosphäre geht einher mit spürbaren Veränderungen des Erdklimas. Langfristszenarien, allen voran der 2018 erschienene Sonderbericht des International Panel on Climate Change (IPCC 2018), führen vor Augen, dass selbst bei Erreichung des $1,5^{\circ} \mathrm{C}$-Ziels, das 2015 im Übereinkommen von Paris formuliert worden ist, irreversible Prozesse losgetreten werden könnten, die unmittelbare wie mittelbare Konsequenzen für menschliche Gesundheit nach sich ziehen. Dazu gehören die Zunahme der Mitteltempera- tur, häufigere und intensivere regionale Hitzeextreme und Starkniederschläge in bewohnten Gebieten sowie regionale Dürren und Niederschlagsdefizite. Hinzu kommt der Anstieg des Meeresspiegels, der mit erhöhtem Salzwassereintrag, Überflutung und Schädigung von Infrastruktur verbunden ist; Biodiversitäts- und Ökosystemverlust durch Erwärmung des klimatisch bestimmenden geografischen Verbreitungsgebiets, Waldbrände oder die Ausbreitung invasiver Arten:

„Jegliche Zunahme der globalen Erwärmung wird sich laut Projektionen auf die menschliche Gesundheit auswirken, mit überwiegend negativen Folgen." (IPCC 2018:13)

Während zu Beginn der Klimaforschung noch auf die reine Beschreibung des Klimasystems und dessen biophysikalische Veränderungen fokussiert wurde, verlagern sich die Forschungsinteressen zunehmend in Richtung der Möglichkeiten gesellschaftlicher Reaktionen auf den Klimawandel. Dabei haben sich zwei grundsätzlich unterscheidbare Prozesse herauskristallisiert: Klimaschutz (Mitigation) und Klimaanpassung (Adaptation). Klimaschutz beschreibt dabei Maßnahmen, die im Wesentlichen auf die Senkung der globalen Durchschnittstemperatur mittels Treibhausgasminderung abzielen. Klimaanpassung dagegen „findet in von Menschen beeinflussten Systemen als Reaktion auf das aktuelle oder erwartete Klima und dessen Folgen statt“ (Marx 2017: 9). Es geht hier folglich um die Bewältigung von Klimafolgen bzw. die Erhöhung der Resilienz gegenüber Klimafolgen. Entscheidender Unterschied zum Klimaschutz besteht darin, dass Anpassungsmaßnahmen einen starken räumlichen Bezug aufweisen und nur in der jeweiligen Umsetzungsregion ihre Wirkung entfalten können. Während die Treibhausgasminderungen durch Klimaschutzmaßnahmen aufgrund der Durchmischung innerhalb der Erdatmosphäre ortsunabhängig wirken, gilt es bei der Klimaanpassung regionale oder lokale Betroffenheiten zu berücksichtigen. 


\subsubsection{Klimawandelbedingte Gesundheits- risiken in deutschen Städten}

Der Klimawandel mit der zu erwartenden $\mathrm{Zu}$ nahme sommerlicher Trocken- und Hitzeperioden, Extremwetterereignissen sowie den sich insgesamt ändernden Temperatur- und Niederschlagsverteilungen wirkt sich direkt wie indirekt auf die menschliche Gesundheit aus .

$\mathrm{Zu}$ den offensichtlichsten klimawandelbedingten Gesundheitsrisiken in der Stadt gehören die Hitzebelastung und daraus resultierender Hitzestress. In Abbildung 1 ist für das Ruhrgebiet die Zunahme von problematischen Stadträumen während Hitzeperioden für das Jahr 2013 und 2100 vergleichend abgebildet, festgestellt anhand von Klimaanalysekarten und einer FITNAH-Modellierung (Flow over Irregular Terrain with Natural and Anthropogenic Heat sources) sowie dem zu erwartenden mittleren Temperaturanstieg (für das Beispiel der Stadt Köln vgl. Kemen et al. 2020).

Heimische Krankheitserreger (Hantaviren, Borrelien übertragen durch Zecken) werden in ihrer Ausbreitung durch ein milderes Klima begünstigt. Ebenso können bisher fremdartige Krankheitserreger (z.B. Dengue- oder Chikungunya-Viren übertragen durch die asiatische Tigermücke) sich so auch in Deutschland ausbreiten (Watts et al. 2019; Umweltbundesamt 2019).

$\mathrm{Zu}$ den vulnerablen Gruppen angesichts langer Hitzeperioden, wie in den Sommern 2018 und 2019, gehören ältere Menschen, Kleinkinder und Säuglinge sowie Menschen mit schweren Vor- und Grunderkrankungen. Aber auch Arbeits- und Wohnbedingungen sind Einflussfaktoren, die die jeweilige Betroffenheit von Hitze mitbestimmen. Berufsgruppen, die einen Großteil ihrer Arbeitsstunden draußen verbringen, sind einer erhöhten Hitzebelastung ausgesetzt; z.B. Dachdecker oder Angehörige der Straßenbaubranche. Wohnungen in höheren Stockwerken, mit schlechter Durchlüftung und/oder Nähe zum Stadtzentrum heizen stärker auf. Das daraus resultierende schlechte Innenraumklima hat negative Aus- wirkungen auf das Wohlbefinden, die Lebensqualität und die Leistungsfähigkeit. Die medizinische Versorgungslandschaft muss sich auf diese Entwicklungen vorbereiten und mit einem erhöhten Patientenaufkommen während sommerlicher Hitzeperioden rechnen.

\section{Hitzebedingte Sterbefälle im Sommer 2018}

\begin{abstract}
Der Sommer 2018 war der zweitheißeste seit Beginn der Wetteraufzeichnung. Auch wenn für Gesamtdeutschland kein gebündeltes Monitoring der Sterblichkeitsdaten existiert, ist zumindest in den Bundesländern Berlin und Hessen ein solches etabliert. Darauf basierend hat das Robert Koch-Institut die hitzebedingten Todesfälle dieses Sommers für Berlin auf 490 geschätzt und für Hessen auf 740. Daraus ergibt sich eine hitzebedingte Mortalität von 12/100.000 Einwohner. In der Altersgruppe der 75- bis 84-Jährigen betrug diese 60/100.000 Einwohner, bei den über 84-Jährigen waren es sogar 300/100.000 (An der Heiden et al. 2019).
\end{abstract}

Darüber hinaus bedarf es in einigen Bereichen angepasster Abläufe und Strukturen: In besonders von Hitze betroffenen Berufsgruppen sollte erwogen werden, Arbeitszeiten und -prozesse den Witterungsbedingungen anzupassen z.B. durch Verlagerung in die kühleren Morgen- und Abendstunden, wie es in heißen Ländern bzw. Regionen schon lange üblich ist.

Gerade im Stadtzentrum wird die Hitzeentwicklung noch befördert durch starke Versiegelung und fehlende Frischluftschneisen, was zu lokalen Wärme- bzw. Hitzeinseln führt. Belastungen auslösen wird zudem die Zunahme von Tropennächten, in denen die Außentemperatur nicht unter $20^{\circ} \mathrm{C}$ sinkt und dadurch den Erholungseffekt des Schlafs hemmt.

\section{Positive Effekte von Dach- und Fassadenbegrünung}

Begrünte Dächer wirken thermisch, lufthygienisch und energetisch auf die jeweilige Gebäudestruktur und potenziell sogar auf das gesamte Mikroklima eines 


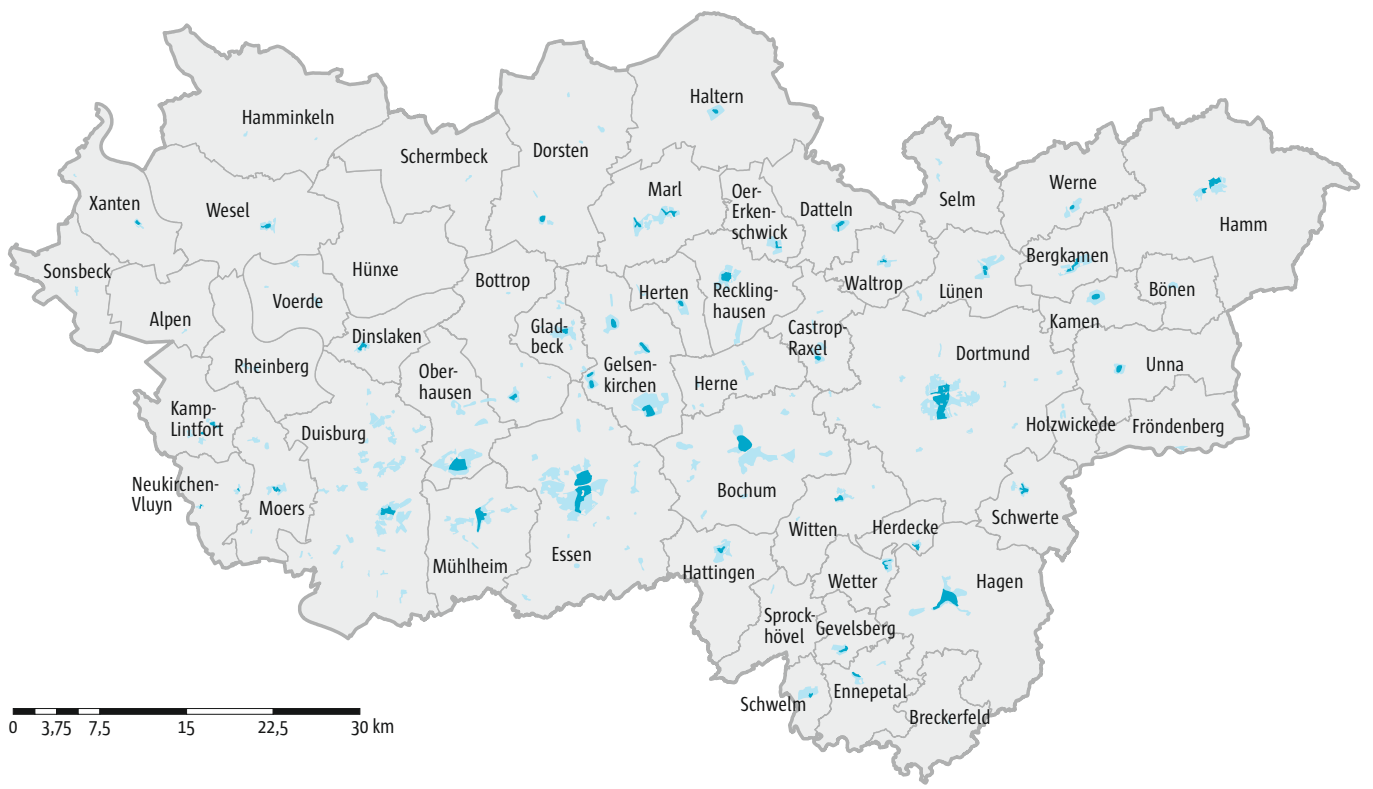

gegenwärtige Problemgebiete (2013)

Wärmebelastung während Hitzephasen: $\square$ hoch $\square$ sehr hoch $\square$ Stadtgrenzen

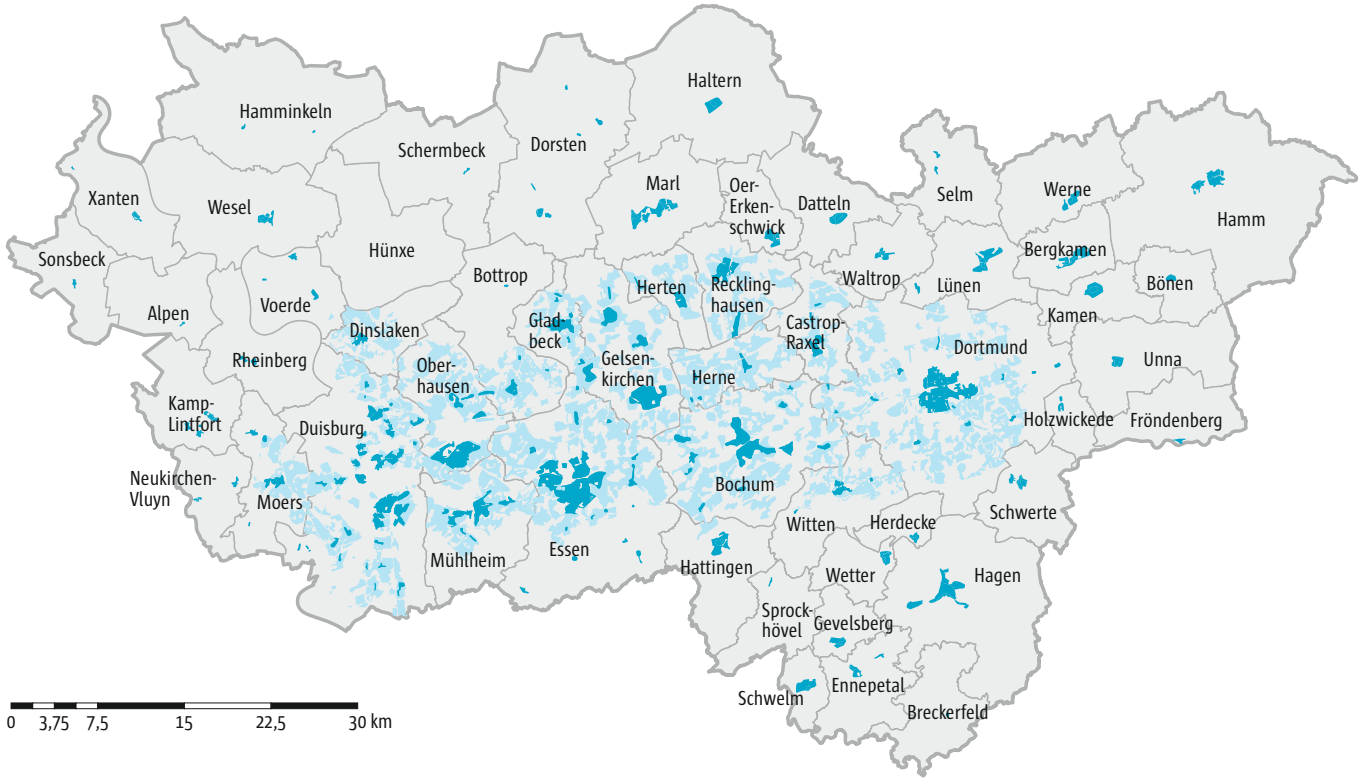

\section{zukünftige Problemgebiete (2100)}

Wärmebelastung während Hitzephasen: $\square$ hoch $\square$ sehr hoch $\square$ Stadtgrenzen

Abb. 1 Gegenwärtige (2013) und zukünftige Problemgebiete (2100) bei Hitzephase in der Metropole Ruhr (RVR 0.J.: S. 78 , mit freundlicher Genehmigung) 
Stadtviertels, sofern Dachbegrünung im größeren Verbund angelegt wird. Es werden Temperaturextreme im Jahresverlauf abgemildert, da Blattwerk, Luftpolster und Verdunstung in der Vegetationsschicht im Sommer die Dachfläche kühlen und im Winter den Wärmeverlust des Hauses mindern. Durch das Auffangen von 70 bis $100 \%$ der Niederschläge in der Vegetationsschicht und anschließender Verdunstung kühlt die Luft in den Stadtteilen ab und Starkniederschläge werden erst zeitverzögert an die Kanalisation abgegeben, wodurch das Stadtentwässerungssystem entlastet wird.

Gleiches gilt für Fassadenbegrünung. Insbesondere der lufthygienische Faktor ist hier zu betonen, da Fassadenbegrünungen Luftverunreinigungen herausfiltern (v.a. Feinstaub), was speziell in engen Straßenschluchten eine wirkungsvolle Begrünungsmaßnahme darstellt (EEA 2020).

Die innerstädtische Versiegelung und Verdichtung beeinflusst zudem die natürliche Bodenstruktur, den Wasserhaushalt und die Wasserinfrastruktur einer Stadt (hier und im Folgenden Mc Call et al. 2020). Durch die verringerten Versickerungsmöglichkeiten wird das Abwasseraufkommen verstärkt und das Risiko von Überflutungen durch Starkregen befördert. Neben dem Risiko von Verschmutzungen und Kontaminationen mit Schadstoffen und Schäden an technischer Infrastruktur können solche Überflutungen und Hochwasser auch die soziale Infrastruktur (z.B. Krankenhäuser) betreffen. Hinzu kommt, dass lokale Unwetter bzw. Extremwetterereignisse wie Starkregen, Hagel, Sturm oder Hochwasser weitere gesundheitsgefährdende Folgen nach sich ziehen können: Gravitative Massenbewegungen (Erdrutsche, Steinschlag), erhöhte Unfallgefahr im Straßenverkehr, verminderte Wasserqualität sowie ebenfalls die Ausbreitung von Tierarten mit Vektorpotenzial (z.B. Mücken, Zecken).

Die bauliche (Nach-)Verdichtung geht oftmals auf Kosten von Grünflächen, wodurch deren temperaturausgleichende und luftverbessernde Effekte verloren gehen. Auch wird der Zugang zu Grünflächen als Bewegungs- und
Aufenthaltsmöglichkeit für die jeweilige Bevölkerung dadurch versperrt. Umfangreiche Analysen sind bereits jetzt notwendig, um wertvolle bestehende klimaökologische Stadtgebiete nicht durch weitere Verdichtung zu zerstören (s. Abb. 2 mit Beispiel Ruhrgebiet).

\subsubsection{Sozial bedingte Betroffenheiten: die Frage der Umweltgerechtigkeit}

Die oben beschriebenen möglichen Risiken und Gesundheitsgefährdungen sind innerhalb der Stadt ungleich verteilt. Ebenso wie umweltbedingte Gesundheitsprobleme aus räumlicher Perspektive erkannt werden können, sind auch soziale und ökonomische Problemlagen räumlich darstellbar. Die Frage, wie gesundheitsrelevante Umweltbelastungen und gesundheitsfördernde Umweltressourcen sozialräumlich verteilt sind, firmiert unter dem Begriff der Umweltgerechtigkeit. „Wesentlich sind hierbei zwei Mechanismen: die soziale Ungleichverteilung von Umweltbelastungen und Umweltgütern (Expositionsvariation) und soziale Unterschiede in der Anfälligkeit (Vulnerabilität) hinsichtlich der gesundheitlichen Wirkungen von Umweltexpositionen (Effektmodifikation)“ (Bolte et al. 2018: 675). Verschiedene Studien haben den Zusammenhang von umweltbedingten (Mehrfach-)Belastungen und sozialer Lage gezeigt (vgl. Bunge $u$. Rehling 2020). Die sozialräumliche Konzentration von Umweltbelastungen (Lärm, Luftschadstoffe, fehlende Grünflächen, schlechte Wohnverhältnisse etc.) korrespondieren mit sozial benachteiligten Stadtquartieren. Charakteristisch ist sowohl das erhöhte Niveau pathogener (Bsp. Luftschadstoffe) als auch das Fehlen salutogener (Bsp. Grünflächen) Umweltfaktoren in solchen Quartieren, die die soziale Vulnerabilität der Bewohner noch weiter erhöhen und sich auf deren allgemeinen Gesundheitszustand auswirken (ebd.). 


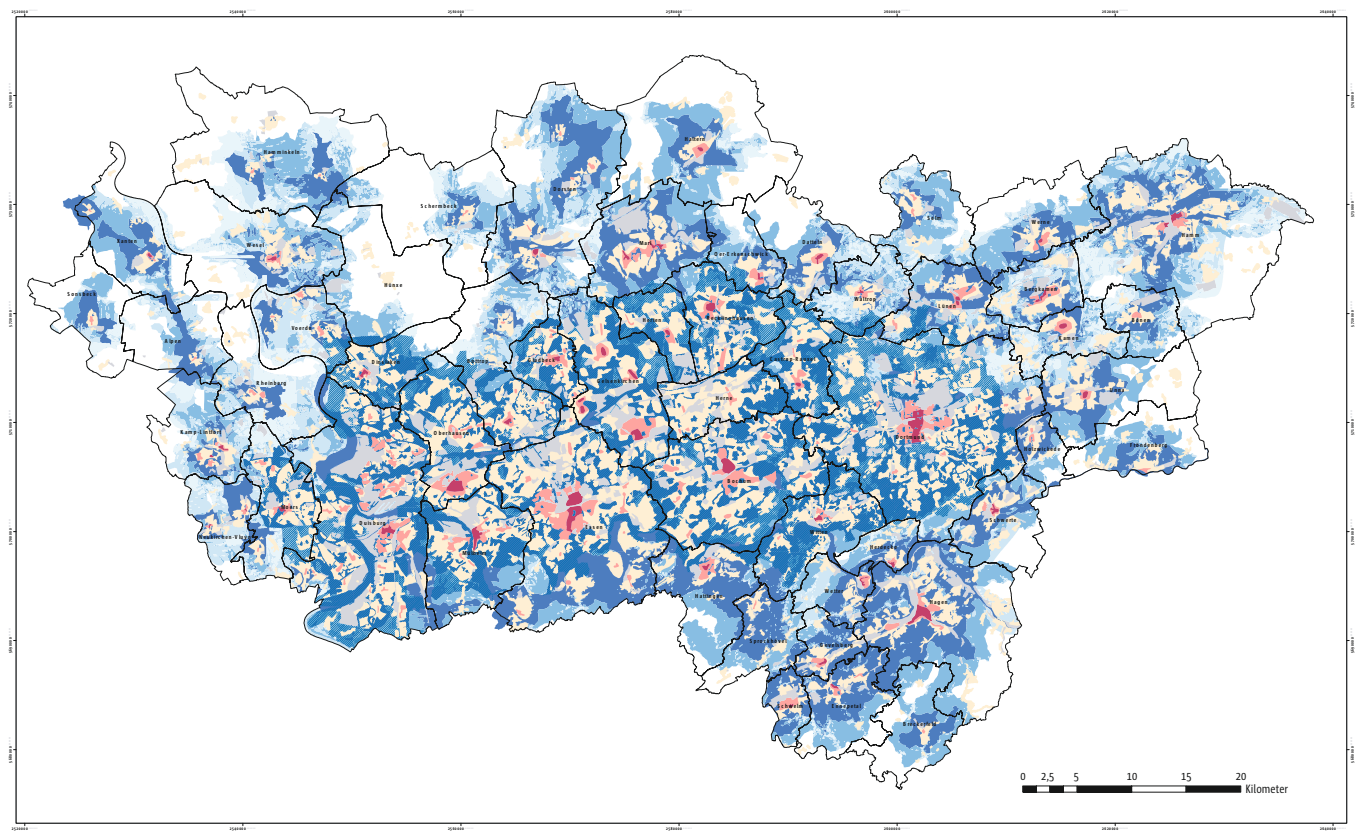

\section{Klimaanpassung in der Metropole Ruhr}

\section{Planungshinweise}

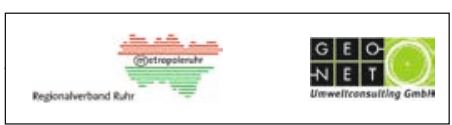

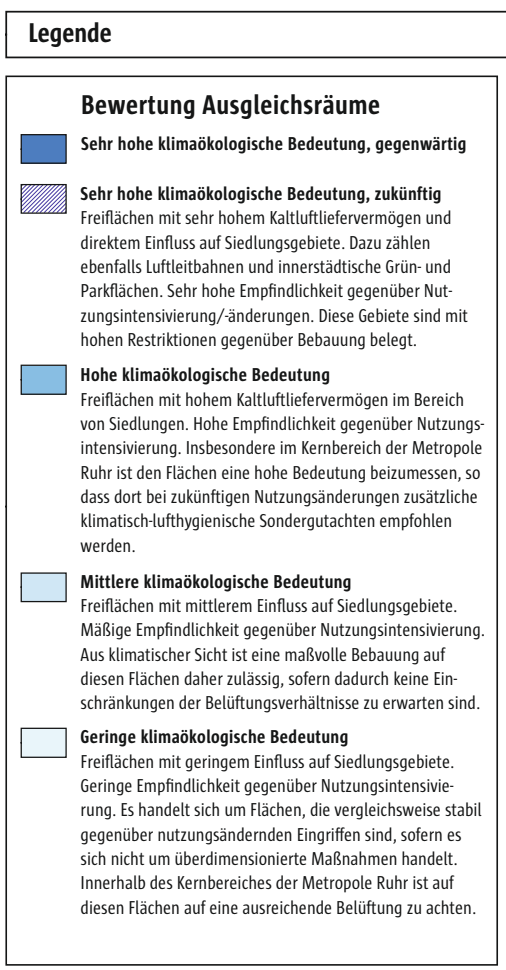

Abb. 2 Bewertung von Ausgleichs- und Lasträumen im Zuge der Klimaanpassung für das Ruhrgebiet (RVR o. J: S. 88, mit freundlicher Genehmigung) 


\section{Zugang zu Grünflächen als gesundheitsfördernder Faktor}

In einer Reihe von wissenschaftlichen Arbeiten wurde ein positiver Zusammenhang zwischen lokalem Zugang zur Natur und reduzierter Morbidität festgestellt, insbesondere bei Herz-Kreislauf- und Atemwegserkrankungen. Während weniger Herz-Kreislauf-Erkrankungen und ein niedrigerer Blutdruck durch Bewegung im Grünen wahrscheinlich sind, wurde auch festgestellt, dass der bloße Kontakt mit der Natur einen positiven Einfluss auf Herzfrequenz und Blutdruck hat. In Bezug auf Typ-2-Diabetes fand man eine geringere Prävalenz bei Menschen, die in der Nähe einer großen Grünfläche leben sowie Zusammenhänge zwischen Grünflächen in der Nachbarschaft und einer geringeren Wahrscheinlichkeit, an Diabetes mellitus Typ 2 zu erkranken (EEA 2020). Eine populationsbasierte Studie im Ruhrgebiet konnte zeigen, dass mit zunehmendem Grün in direkter Wohnumgebung sowohl der Gesundheitszustand positiver bewertet wurde als auch die Zufriedenheit mit der Nachbarschaft und das Sicherheitsempfinden anstiegen - unabhängig von Sozialschicht und weiteren Einflussfaktoren (Orban et al. 2017).

Die Betroffenheit einzelner Städte vom Klimawandel hängt somit zum einen von den konkreten klimatischen Parametern vor Ort sowie von den jeweiligen Merkmalen und Prozessen ab, die die ökonomischen und sozialen Schäden und deren gesellschaftliches Bewältigungspotenzial bestimmen. Bei der Abschätzung und Beurteilung möglicher Klimaschäden und -folgen gilt es, das komplexe Netz aus sozialen, ökonomischen, ökologischen und physisch-infrastrukturellen Faktoren als Ganzes zu betrachten und darauf aufbauend Städte zu klimagerechten, gesundheitsfördernden und lebenswerten Orten zu machen.

\subsection{Ansatzpunkte einer integrierten klimasensiblen Stadtentwicklung zur Gesundheitsförderung}

Die aktuelle und künftige Lebensqualität der Bewohner einer Stadt hängt somit wesentlich von klimaverträglichen und klimaresilienten Konzepten der Stadtentwicklung ab. Räumliche Strukturen einer Stadt können durch Art und Gestaltung der baulichen Nutzung die klimatische Situation entscheidend beeinflussen und gestalten und dadurch direkt oder indirekt Einfluss auf menschliche Gesundheit nehmen. Mögliche Konzepte urbaner Transformation sind die postfossile und die resiliente Stadt. Die Herausforderung einer postfossilen Stadt besteht in der erfolgreichen Transformation von einer auf fossilen Energien und hohem Energieverbrauch basierenden Stadt hin zu einer Stadt mit niedrigem Energieverbrauch unter Nutzung erneuerbarer Energien. Eine solche Transformation impliziert massive Eingriffe in nahezu sämtliche kommunale Politikfelder und umfasst technologische, ökonomische und soziale Innovationen hinsichtlich Energie-, Wärmeund Kälteproduktion sowie deren Verteilung und Konsumtion (Rink u. Kabisch 2017: 255). Das betrifft den Verkehrs- ebenso wie den Bauund Sanierungsbereich.

Die Transformation zur resilienten Stadt zielt derweil auf die Vulnerabilität von Städten gegenüber Klimaänderungen und deren Folgen ab und konzentriert sich auf mögliche bzw. notwendige Anpassungsmaßnahmen. „Zu prüfen ist vor allem, wie das Konzept der resilienten Stadt praktische Relevanz für Planer und die kommunale Verwaltung oder Katastrophenschutzorganisationen und - agenturen gewinnen kann, um Vorsorge- und Reaktionskapazitäten in Städten bzw. Siedlungsbereichen aufzubauen und zu verbessern" (Rink u. Kabisch 2017: 258). Dies betrifft vor allem die Kommunen als Umsetzungsadressaten, denen es jedoch in den meisten Fällen an ausreichend finanziellen und personellen Ressourcen mangelt, insbesondere für eigene stadtklimatische Untersuchungen. Im Juni 2020 hat das Landesamt für Natur, Umwelt und Verbraucherschutz NRW (LANUV) gemeinsam mit dem Deutschen Wetterdienst und der Stadt Bonn ein OnlineTool für Kommunen und Stadtplaner entwickelt, das die Prüfung der Wirkung verschiedener Anpassungsmaßnahmen im Stadtquartier 
ermöglichen soll (https://www.klimaanpassung-karte.nrw.de/?feld=inkas-nrw).

Bei beiden Konzepten müssen immer die komplexen Wirkungszusammenhänge beachtet und darauf aufbauend ganzheitliche Lösungsansätze statt isolierter Einzelmaßnahmen angestrebt werden. „Pauschale Aussagen über die Auswirkungen von planerischen Eingriffen auf das gesamte Stadtgefüge sind nicht möglich. Vielmehr gilt es, die Faktoren bei jedem Projekt ganzheitlich zu bewerten und je nach Situation und Standort abzuwägen" (Anders 2018: 31). Dabei sind postfossile und resiliente Strategien nicht trennscharf voneinander zu separieren, sondern weisen Schnittmengen auf und sollten entsprechend kombiniert gedacht werden.

Grundsätzliche Interventionsmöglichkeiten, um zunehmender Hitzebelastung in Städten entgegenzuwirken, ist die Nutzung des thermisch dämpfenden Potenzials von Grünund Wasserflächen, also die Schaffung von stadtblauen und -grünen Elementen (Kemen et al. 2020). Überwiegend grüne oder blaue, wenig versiegelte Flächen übernehmen wichtige Funktionen für das lokale Kleinklima. Die positive Wirkung von Grünflächen auf das Stadtklima sowie die Luftqualität und Lärmminderung ist abhängig von Größe, Aufbau und Zusammensetzung der vegetationsbestandenen Fläche. Mit Gras bewachsene Flächen wirken positiv auf die Strahlungs- und Wärmebilanz, während durch Bäume und Sträucher erzeugte Schattenplätze bioklimatische Effekte weiter ergänzen. An Kaltluftentstehungsgebiete des ländlichen Umlands (Wiesen und Felder) sind diese städtischen Grünflächen idealerweise über Ventilationsbahnen angebunden. Die Ausweisung großräumiger Freiflächen als Vorrang- und Vorbehaltsgebiete für besondere Klimafunktionen (insbesondere mit Blick auf die Luftzirkulation in einem Stadtquartier) und deren Vernetzung mit lokalen Grünflächen ist hier als raumplanerisches Instrument möglich.

Neben den thermischen Effekten ist insbesondere Stadtblau für die psychische Gesundheit und das Wohlbefinden bemerkenswert, insofern es einen positiven Zusammenhang gibt zwischen der Exposition gegenüber blauen Räumen und der psychischen Gesundheit, dem Wohlbefinden und dem Grad der körperlichen Aktivität (Gascon et al. 2017). Es zeigt sich zudem, dass das Wohlbefinden von Stadtbewohnern besonders mit blauen Räumen assoziiert wird, und zwar durch verstärkte Kontemplation, emotionale Bindung, Teilnahme und körperliche Aktivität (Völker u. Kistemann 2011).

Um die Hitze in Innenräumen zu reduzieren, sind Maßnahmen der thermischen Gebäudeisolation, der Dach- und Hausbegrünung sowie der hitzeadäquaten Gebäudeplanung Ansatzpunkte direkt am Gebäude selbst; sowohl bei der Sanierung des Gebäudebestands als auch beim Neubau. Solch bauliche Maßnahmen betreffen auch die Planung und Gestaltung von Krankenhäusern und Pflegeeinrichtungen, um sowohl die Energiebilanz der Gebäudekomplexe zu steigern und damit aktiven Klimaschutz zu betreiben als auch durch die Maßnahmeneffekte der Klimaanpassung die Bedingungen für Patienten und Personal zu verbessern:

\begin{abstract}
Überlegenswert sind zudem neue Konzepte von Gesundheitsversorgung, die aktiv in die Gestaltung der Megatrends des 21. Jahrhunderts - Urbanisierung, Klimawandel und Digitalisierung - einbezogen und integriert werden. Ein Ansatz könnte dabei sein, das Smart Hospital jenseits der Begrenzung durch Klinikmauern zu denken und Gesundheitsversorgung integrativ über die verschiedenen Räume einer Stadt (Wirtschafts-, Bildungs-, Sozial-, Kultur-, Umwelt-, Verkehrsraum) zu entfalten. Gesundheitsversorgung wird dadurch quer durch alle Stadträume mitgedacht und kann Entwicklungsimpulse geben.
\end{abstract}

Mit Blick auf die sich möglicherweise ändernde Häufigkeit und Intensität von Hochwasserereignissen, ist ein vorausschauernder und vorbeugender Hochwasserschutz eine wichtige Klimafolgenanpassungsmaßnahme. Vor allem die Erfassung der betroffenen Überschwemmungsgebiete, die bei einem hundertjährlichen Hoch- 
wasserereignis überflutet würden sowie die Identifizierung und Ausweisung von Gebieten zur Hochwasserentlastung und Rückhaltung sind hier als raumordnerisches Instrument von Bedeutung. Aber auch angesichts kurzfristiger Überflutungsrisiken durch Starkregen bedarf es der Sicherung vorhandener bzw. Schaffung neuer Abfluss- und Retentionsflächen.

Dies sind nur einige Beispiele wie Stadtplanung und Stadtentwicklung auf Klimaschutz und Klimafolgenanpassung Einfluss nehmen und diese sogar aktiv mitgestalten können. Neben der Verhinderung von Risiken sollte hier auch verstärkt der Blick auf die Ausgestaltungsmöglichkeiten von Ressourcen gerichtet und bewusst eine salutogenetische Perspektive eingenommen werden. Denn es ist in vielerlei Hinsicht bis heute unklar „wie guter, gesundheitsförderlicher öffentlicher Raum (Plätze, Straßen) beschaffen sein muss in Bezug aufZugang, Umwelt- und Aufenthaltsqualität, soziale Kontaktmöglichkeiten, partizipative Gestaltungsmöglichkeiten - und diese jeweils mit Blick auf spezifische Bevölkerungsgruppen (Geschlecht, Soziodemografie, Lebensphase, Migration, Arbeit, Krankheit)“"(Moebus 2020: 11).

\subsection{Umsetzungsbeispiele}

\subsubsection{Lebenswerte Straße in resilienten Quartieren}

Die Gestaltung der gebauten Stadt sollte in diesem Sinne die Dimensionen Dekarbonisierung, systemische Resilienz, grüne Infrastrukturen sowie soziale Integration und Begegnung als Ziel integrativ betrachten. Erste Ansätze finden sich in einzelnen Pilotprojekten, die versuchen einen Paradigmenwechsel bisheriger Stadtplanung herbeizuführen. $\mathrm{Zu}$ nennen sind hier u.a. die Städte Kopenhagen und Wien.

Konkrete Planungsentwürfe für den Umbau von zwei Quartiersstraßen im Ruhrgebiet werden im Projekt LesSON des Wuppertal Instituts angefertigt. Dabei sollen nationale und internationale Trends der Stadtplanung und des
Stadtumbaus aufgegriffen und an den ökologischen Herausforderungen wie Klimawandel, Klimaanpassung und Biodiversität ausgerichtet werden und dabei gleichzeitig die Bedarfe der Bewohner der umzugestaltenden Quartiersstraßen in den Vordergrund gestellt werden (Wuppertal Institut 2020).

\subsubsection{Zukunftsinitiative „Wasser in der Stadt von morgen"}

In der Zukunftsinitiative „Wasser in der Stadt von morgen“ haben Kommunen der Emscherregion (Ruhrgebiet) sich als Emschergenossenschaft zusammengeschlossen, um die fachübergreifende Zusammenarbeit innerhalb und zwischen den kommunalen Verwaltungen voranzubringen (http://www.wasser-in-der-stadt. de/wasser-in-der-stadt-von-morgen/). Akteure aus Fach- und Verantwortungsbereichen, wie Wasserwirtschaft, Umwelt, Verkehr, Stadtund Freiraumentwicklung zielen dabei auf fachübergreifende Kooperationen und Planungen. Im Zentrum steht die Rolle von Wasser in der Stadtgestaltung und für die Klimaanpassung. Dies umfasst u.a. die Vernetzung von Grünzügen und Wasserachsen, die Schaffung temperaturregulierender Wasserflächen, dezentrale Puffer- und Speicherräume zum Rückhalt von Starkregen und die Gestaltung urbaner Landschaft mittels Bewirtschaftung durch Regenwasser.

In ihrem 2019 fortgeschriebenen Maßnahmenplan 2020+ (Zukunftsinitiative 2019) setzt sich die Zukunftsinitiative für die gesundheitsförderliche Entwicklung ihrer Städte mittels einer integrierten Gesundheits-, Sozial- und Umweltberichterstattung ein, um so insbesondere Stadtgebiete mit Mehrfachbelastungen und erhöhten Handlungsbedarfen zu identifizieren. Zudem sollen kommunale Gesundheitskonferenzen zum Wissenstransfer und zur Sensibilisierung für das Potenzial grüner und blauer Infrastrukturen dienen und relevante Akteure vernetzten, um die Zusammenarbeit zu stärken. 


\subsection{Abschlussbemerkungen}

Die oben aufgeführten Ansätze zeigen, dass eine ganzheitliche Stadt- und Quartiersentwicklung einen wichtigen Beitrag zur urbanen Gesundheitsförderung leisten kann bzw. die Perspektive der Gesundheitsförderung in der Entwicklung von Stadt- und Quartierskonzepten fruchtbare Impulse gibt. Dennoch muss dieser Aspekt noch prominenter herausgestellt und in den verschiedenen politischen Ebenen verankert werden. Wichtiger Baustein dazu ist ein Paradigmenwechsel von einem pathogenen zu einem salutogenen Gesundheitsbegriff zur Beantwortung der Frage, wie Städte in Zukunft gestaltet sein sollen bzw. müssen, um nachhaltiges und gesundes Leben möglich zu machen.

Der WBGU identifiziert hierzu acht Schwerpunkte im Feld urbaner Gesundheit, die bisher zu stark vernachlässigt wurden, zukünftig jedoch von steigender Bedeutung sind (WBGU 2016: 23):

- globaler Paradigmenwechsel von Krankheitsbekämpfung zu Gesundheitsförderung durch Stärkung von Ressourcen und Potenzialen für ein gesundes Leben in Städten

- Gesundheitsförderung durch sektorübergreifende Stadtplanung bzw. -entwicklung und Stärkung kommunaler Planungskonzepte dauerhaft verankern

- Gesundheitskompetenz und -handeln der Stadtbevölkerung fördern

- substanzielle Teilhabe sichern und Nahversorgung verbessern

- Städte gesundheitsfördernd gestalten mit Fokus auf Begegnungs- und Aktivitätsräumen

- Selbstorganisation von Stadtbewohnerinnen stärken, kleinräumige gesundheitsfördernde Maßnahmen im Quartier ermöglichen

- urbane Epidemien und neue Infektionskrankheiten durch Förderung der Resilienz der Bevölkerung, Gesundheitsbildung und Verbesserung der Gesundheitsberichterstattung eindämmen
- Gesundheitsförderung durch sektorübergreifende Stadtplanung (Synergien Klimaschutz/Dekarbonisierung) anstreben

Gerade die Corona-Krise hat vor Augen geführt, von welcher Bedeutung das unmittelbare Lebensumfeld ist, wie z.B. die Wiederentdeckung lokaler Solidarität und Wirtschaftsstrukturen oder auch die Qualität von Erholungsräumen in unmittelbarer Nähe. Eindrücklich waren die Bilder von verwaisten Innenstädten einerseits und belebten Grünflächen andererseits. Dies zeigt, dass viele unserer Innenstädte offenbar über zu wenig Aufenthaltsqualität verfügen und abseits des Konsums kein Anreiz besteht, um sich dort aufzuhalten. Auch sind durch die Corona-Pandemie bereits bestehende Ungleichheiten nochmals deutlich hervorgetreten: beengte Wohnverhältnisse, prekäre Arbeitsbedingungen und Einkommensausfälle, kein $\mathrm{Zu}$ gang zu Stadtgrün/-blau sind nur einige wenige Beispiele. Schneidewind et al. plädieren angesichts dessen dafür, Lehren aus der Corona-Krise zu ziehen:

„Die Innenstadt der Zukunft darf nicht allein Einkaufort sein. Sie muss multifunktionaler werden. Sie muss Arbeits-, Wohn-, Begegnungs-, Lern-, Spiel-, Betreuungs-, Logistik-, Gastronomie- und Einkaufsmöglichkeiten in kluger Weise miteinander kombinieren. [...] Wenn Innenstädte dann auch noch grüner werden als sie derzeit meistens sind, dazu eine andere Mobilität aufweisen, mit weniger motorisiertem Individualverkehr, einem optimierten ÖPNV mit Schnittstellen zu Car- und Fahrrad-SharingAngeboten, werden sie zu Erholungs-, Erlebnis- und Außenräumen mit Aufenthaltsqualität, die Menschen auch außerhalb der Ladenöffnungszeiten nutzen können." (Schneidewind et al. 2020: 6f)

Angesichts der zu erwartenden Klimaänderungen sieht sich der Gesundheitssektor einem erhöhten Versorgungsbedarf gegenüber, unter anderem bezogen auf Hitzestress, Luftqualität sowie Ausbreitung von Krankheiten. Eine klimasensible Stadtplanung, die Gesundheitsförderung explizit in ihre Agenda aufnimmt und 
in Verwaltungs- und Bürgerhandeln integriert, kann wesentlich dazu beitragen, diesen Versorgungsbedarf zu senken.

\section{Literatur}

An der Heiden M, Buchholz U, Uphoff H (2019): Schätzung der Zahl hitzebedingter Sterbefälle und Betrachtung der Exzess-Mortalität; Berlin und Hessen, Sommer 2018. Epidemiologisches Bulletin Nr. 23. Berlin.

Anders S (2018): Mehrwert nachhaltiger Stadtquartiere. In: Bott H, Grassl GC, Anders S (Hrsg.) (2018): Nachhaltige Stadtplanung. Lebendige Quartiere, Smart Cities, Resilienz. DETAIL Business Information $\mathrm{GmbH}$, München. S. 28-31.

Baumgart S (2018): Räumliche Planung und öffentliche Gesundheit - eine historische Verknüpfung. In: Baumgart $S$, Köckler H, Ritzinger A, Rüdiger A (Hrsg.) (2018): Planung für gesundheitsfördernde Städte. Hannover, Forschungsberichte der ARL 08, S. 20-36.

Bolte G, Bunge C, Hornberg C, Köckler H (2018): Umweltgerechtigkeit als Ansatz zur Verringerung sozialer Ungleichheiten bei Umwelt und Gesundheit. Bundesgesundheitsblatt, 61. Jahrgang, Heft 6, S. 674-683.

Bunge C, Rehling I (2020): Umweltgerechtigkeit in Städten. Empirische Befunde und Strategien für mehr gesundheitliche Chancengleichheit. In: Bundesinstitut für Bau-, Stadt- und Raumforschung (BBSR) im Bundesamt für Bauwesen und Raumordnung (BBR) (Hrsg.) (2020): Gesundheit und Krankheit in räumlicher Perspektive. Informationen zur Raumentwicklung, Heft 1/2020. Stuttgart, Franz Steiner Verlag. S. 70-83.

EEA - European Environment Agency (2020): Healthy environment, healthy lives: how the environment linfluences health and well-being in Europe. EEA Report No. 21/2019. Luxembourg, Copenhagen.

Gascon M, Zijlema W, Vert C, White MP, Nieuwenhuijsen, MJ (2017): Outdoor blue spaces, human health and well-being: A systematic review of quantitative studies. International Journal of Hygiene and Environmental Health, Volume 220, Issue 8, S. 1207-1221.

Howard E (2010 [1989]): Tomorrow a Peaceful Path to Real Reform. New York: Cambridge University Press, New York.

IPCC - Intergovernmental Panel in Climate Change (2014): Climate Change 2014: Synthesis Report. Contribution of Working Groups I, II and III to the Fifth Assessment Report of the Intergovernmental Panel on Climate Change. (Core Writing Team: R.K. Pachauri and L.A. Meyer (eds.)). IPSS, Geneva, Switzerland.

IPCC - Intergovernmental Panel in Climate Change (2018): Global Warming of $1.5^{\circ} \mathrm{C}$. An IPCC Special Report on the impacts of global warming of $1.5^{\circ} \mathrm{C}$ above proe-industrial levels and related global greenhouse gas emission pathways, in the context of strengthening the gobal response to the threat of climate change, sustainable development, and efforts to eradicat poverty. (Masson-Delmotte, V.; P. Zhai; H.-O. Pörtner;
D. Roberts; I. Skea; P.R. Shukla; A. Pirani; W. Moufouma-Okia; C. Péan; R. Pidcock; S. Conners; I.B.R. Matthews; Y. Chen; X. Zhou; M.I. Gomis; E. Lonnoy; T. Maycock; M. Tognos; and T. Waterfield (eds.)). World Meteorological Organization, Geneva, Switzerland.

Kautnek T (2012): Der Clean Development Mechanism als Regulativ postfordistischer Naturverhältnisse. Zum Spannungsverhältnis zwischen Kosteneffizienz und nachhaltiger Entwicklung. Diplomarbeit. Universität Wien, Wien. Philologisch-Kulturwissenschaftliche Fakultät.

Kemen I, Schäffer-Gemein S, Kistemann, T (2020): Klimaanpassung und Hitzeaktionspläne. Ein idealtypisches Thema der geografischen Gesundheitsforschung. In: Bundesinstitut für Bau-, Stadtund Raumforschung (BBSR) im Bundesamt für Bauwesen und Raumordnung (BBR) (Hrsg.) (2020): Gesundheit und Krankheit in räumlicher Perspektive. Informationen zur Raumentwicklung, Heft 1/2020. Stuttgart, Franz Steiner Verlag. S. 58-69.

Lorberg D, Simon K (2020): Engels und die Stadt. In: Lucas R, Pfriem R, Westhoff H-D (Hrsg.): (2020): Arbeiten am Widerspruch - Friedrich Engels zum 200. Geburtstag. Marburg. Metropolis-Verlag. S. 365-396.

Marx A (2017): Klimawandel - ein Überblick. In: Marx A (Hrsg.) (2017): Klimaanpassung in Forschung und Politik. Wiesbaden. Springer Spektrum. S. 3-16.

Mc Call T, Liedtke TP, Liebig-Gonglach M, Freymüller I, Hornberg C (2020): EcoHealth und Stadtplanung. Eine Public-HealthPerspektive. In: Bundesinstitut für Bau-, Stadt- und Raumforschung (BBSR) im Bundesamt für Bauwesen und Raumordnung (BBR) (Hrsg.) (2020): Gesundheit und Krankheit in räumlicher Perspektive. Informationen zur Raumentwicklung, Heft 1/2020. Stuttgart, Franz Steiner Verlag. S. 84-95.

Meadows DH, Meadows DL; Randers, Jørgen; Behrens III, William W (1972): The limits to growth. A report for the Club of Rome's project on the predicament of mankind. New York: Universe Books.

Moebus S (2020): Gebaute Stadt und Gesundheit - Bedeutung und Implikationen für die Prävention und Gesundheitsförderung. In: Tiemann M, Mohokum M (Hrsg.) (2020): Prävention und Gesundheitsförderung. Springer Reference Pflege - Therapie - Gesundheit.

Orban E, Sutcliffe R, Dragano N, Jöckel K-H, Moebus S (2017): Residential Surrounding Greenness, Self-Rated Health and Interrelations with Aspects of Neighborhood Environment and Social Relations. J Urban Health. 94(2):158-169

Regionalverband Ruhr (RVR) (o.A.J.). Fachbeitrag zum Regionalplan der Metropole Ruhr „Klimaanpassung“. Regionalverband Ruhr Referat Geoinformation und Raumbeobachtung Referat Team Klimaschutz, Klimaanpassung und Luftreinhaltung. Essen. Zugriff 29.10.2020: www.rvr.ruhr/fileadmin/ user_upload/01_RVR_Home/02_Themen/Regionalplanung_ Entwicklung/Regionalplan_Ruhr/04_Fachbeitraege/Fachbeitrag_Klimaanpassung.pdf

Rink D, Kabisch S (2017): Urbane Transformationen und die Vision nachhaltiger Stadtentwicklung. In: Brand, Karl-Werner (Hrsg.) (2017): Die sozial-ökologische Transformation der Welt. Ein Handbuch. Campus Verlag, Frankfurt/New York, S. 243-266. 
Rodenstein M (1988): „Mehr Licht, mehr Luft“: Gesundheitskonzepte im Städtebau seit 1750. Frankfurt a.M., New York. Campus Verlag. Zugleich: Berlin, Technische Universität, Habilitationsschrift.

Rosenbaum W, Mautz R (2011): Energie und Gesellschaft: Die soziakle Dynamik der fossilen und der erneuerbaren Energien. In: Matthias Groß (Hrsg.): Handbuch Umweltsoziologie. 1. Auflage Wiesbaden: VS Verlag für Sozialwissenschaften, S. 399-420.

Schneidewind U, Baedeker C, Bierwirth A, Caplan A, Haake H (2020): „Näher“ - „Öffentlicher“ - „Agiler“. Eckpfeiler einer resilienten „Post-Corona-Stadt“. Diskussionspapier. Wuppertal Institut.

Watts $\mathrm{N}$ et al. (2019): The 2019 report of The Lancet Countdown on health and climate change: ensuring that the health of a child born today is not defined by a changing climate. Lancet Vol. 394, Issue 102011, S. 1836-1878.

Umweltbundesamt (Hrsg.) (2019): Monitoringbericht 2019 zur Deutschen Anpassungsstrategie an den Klimawandel. Bericht der interministeriellen Arbeitsgruppe Anpassungsstratgie der Bundesregierung. Dessau-Roßlau.

United Nations, Department of Economic and Social Affairs, Population Division (2019): World Urbanization Prospects: The 2018 Revision (ST/ESA/SER.A/420). New York: United Nations.
Vereinte Nationen (2015): Transformation unserer Welt: die Agenda 2030 für nachhaltige Entwicklung. Resolution der Generalversammlung, verabschiedet am 25. September 2015. A/ RES/70/1. New York.

Völker S, Kistemann T (2011): The impact of blue space on human health and well-being - Salutogenetic health effects of inland surface waters: A review. International Journal of Hygiene and Environmental Health, Volume 214, Issue 6, S. 449-460.

WBGU - Wissenschaftlicher Beirat der Bundesregierung Globale Umweltveränderungen (2011): Welt im Wandel. Gesellschaftsvertrag für eine Große Transformation. Berlin WBGU.

WBGU - Wissenschaftlicher Beirat der Bundesregierung Globale Umweltveränderungen (2016): Der Umzug der Menschheit: Die transformative Kraft der Städte. Berlin. WBGU.

Wuppertal Institut (Hrsg.) (2020): „Lebenswerte“ Straße in resilienten urbanen Quartieren. Projektergebnisse eines Teilprojektes im Gesamtprojekt „Eckpunkte für die Umsetzung einer Landesstrategie zur Klimaanpassung aus wissenschaftlicher Sicht“. Wuppertal Report 17. Wuppertal.

Zukunftsinitiative (2019): Maßnahmenplan 2020+ der Zukunftsinitiative „Wasser in der Stadt von morgen“. Fortschreibung 2019. http://www.wasser-in-der-stadt.de/fileadmin/Medien/Projekte/Dokumente/20190521_Entwurf_Aktualisierung_2019_Gesamtdokument.pdf

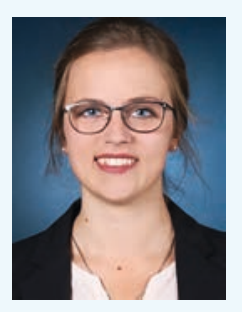

Judith Schröder, M.A.

Judith Schröder studierte Philosophie, Politik- und Europawissenschaften und war mehrere Jahre am Wuppertal Institut für Klima, Umwelt, Energie im Forschungsfeld der Energie- und Klimapolitik tätig bevor sie ans Institut für Urban Public Health am Universitätsklinikum Essen kam, um die Zusammenhänge von ökologischer Krise, Stadtentwicklung und Urban Public Health zu beforschen.

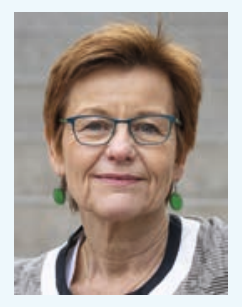

Prof. Dr. rer. nat. Susanne Moebus, MPH

Studium der Biologie in Bremen und Public Health in Bielefeld. Professorin für Urbane Epidemiologie am Universitätsklinikum Essen der Universität Duisburg-Essen und Leiterin des Zentrums für Urbane Epidemiologie am Institut für Medizinische Informatik, Biometrie und Epidemiologie seit 2012. Anfang 2020 Leitung des neu gegründeten Instituts für Urban Public Health (InUPH) am Universitätsklinikum Essen. Neben bevölkerungsbezogener epidemiologischer Forschung im Rahmen von großen Kohortenstudien liegen Schwerpunkte ihrer interdisziplinären und anwendungsorientierten Forschung in der Beschreibung und Analyse gesundheits- und krankheitsbezogener Zusammenhänge in städtischen Räumen. 